\title{
Numerical study on conjugate convective thermal transport in an annular porous geometry
}

\author{
S. Kiran ${ }^{1}$, M. Sankar ${ }^{2}$, S. Sivasankaran ${ }^{3, *}$ \\ ${ }^{1}$ Dept. of Mathematics, Nitte Meenakshi Institute of Technology, Bengaluru, Karnataka, India \\ ${ }^{2}$ General Requirements Department, University of Technology and Applied Sciences - \\ Ibri, Ibri 516, Oman \\ ${ }^{3}$ Dept. of Mathematics, King Abdulaziz University, Jeddah, Saudi Arabia \\ *Corresponding author: sd.siva@yahoo.com
}

\begin{abstract}
Buoyancy-driven convection in an annular space between two upright concentric cylinders having a finite thickness of inner/outer cylinder is an essential physical structure exposing several practical applications. The current article reports the coupled conduction-convection transfer in an upright porous annular space and the buoyant convective stream and thermal transfer, associated thermal transport rates has been numerically investigated. In this analysis, the inner cylinder has a fixed width and is maintained at a uniform high temperature, while the outer cylinder wall is preserved at a uniform lower temperature. However, the lower \& upper boundaries of the annular region are presumed to be sealed and insulated. The Brinkman-extended Darcy formulation is implemented for modeling the stream in the porous medium. An implicit finite difference technique based on SLOR \& ADI methods is adopted to resolve the governing equations. From the numerical predictions, it has been detected that the conductivity ratio \& wall thickness has a crucial role in controlling thermal transport through the annular space. The present work will have applications in electronic equipment, electric machinery, solar collectors, and lubrication systems.
\end{abstract}

Keywords: Annular enclosure; conjugate heat transfer; Darcy-Brinkman model; finite difference method porous.

\section{Introduction}

The exploration of free convection in an annular chamber has been analyzed by various investigators due to its importance in numeral natural phenomena and in industrial applications, such as cooling of electronic chips, energy conversion exchanges of heat energy through multishell packaging, building comfort, transmission \& storage systems, and other alternative fields, Kiwan and Zeitoun, (2008), AL-Jawary, (2020), Al-Ashhab, (2019). A significant amount of theoretical and experimental mechanisms is directed in the past periods in an endeavor to grasp heat transfer flow in a cavity Ghalambaz et al. (2019), Nazarafkan et al. (2019). The first extensive numerical investigation on buoyant convection in an upright annular space with differently heated (vertical) cylinders and adiabatic horizontal boundaries is by de Vahl Davis and Thomas (1969). They established a relationship for the averaged thermal transport in various stream regimes. 
Further, the influence of several parameters like aspect ratio and the radius ratio of the annular geometry on convective thermal transport is carried out in the literature (Sankar and Do, (2010), Massarotti et al. (2016)). Later, the discrepancies that exist in the relation established in de Vahl Davis and Thomas (1969) have been found, and a novel correlation has been developed for global heat transfer rates covering a more comprehensive range of $\mathrm{A}, \lambda$, and Ra. Venkatachalappa et al. (2001) performed numerical simulations in an annulus, with rotating inner and outer walls and observed a higher heat transfer rate for turning walls compared to stationary boundaries. MebarekOudina (2017) presented the numerical results on hydrodynamic stability in an annulus having thermal sources of different lengths. The buoyant convection heat transfer caused by discrete heating of the inner wall of an upright cylindrical annulus is investigated by Sankar and co-workers Lopez et al. (2012), Sankar et al. (2015).

Buoyant convection in a porous medium has attracted many theoretical and experimental researchers through decades due to its strong involvement in many engineering applications, in particular, porous annular geometry with numerous thermal conditions has received substantial attention in the literature. A detailed review of existing flow models in various geometries accounting for different constraints is well documented in many research articles Bourouis et al. (2016), Hdhiri and Ben Beya, (2018), Ayoubloo et al. (2019). The above literature reviews explore many experimental and numerical investigations to study the fluid stream and heat energy transference in porous media. The pioneering investigations on convective transport processes in a porous annulus are due to Prasad and co-workers Prasad and Kulacki (1984), Prasad (1986), Hickox and Gartling (1985), Havstad and Burns (1982), and Hasnaoui et al. (1995). These studies spanned over different porous media models and covered a vast range of radius and aspect ratios and other physical parameters and proposed thermal transport correlations. Shivakumara et al. (2002) executed a theoretical analysis of buoyance convection in a porous annular space. Sankar and co-workers Sankar et al. (2011), Sankar et al. (2011), Sankar et al. (2013) performed numerical predictions on the buoyant stream and thermal transfer characteristics from discrete heating in square and annular configurations packed with a fluid-saturated porous material. The main objective in their simulations was to identify an optimum location of the thermal source to maximize the thermal transport in the chosen geometry. The buoyant thermal transport rates are highly sensitive to the thermal profiles applied on the enclosure walls. It has been demonstrated through numerical predictions that sinusoidal thermal profiles significantly change the heat transfer characteristics in a square geometry compared to uniform thermal conditions Sivasankaran and Bhuvaneswari (2013), Wu et al. (2015).

Buoyant heat transport in finite geometries is influenced by numerous components, and indeed the important amongst these is the situation when one of the rigid walls with finite width. The presence of the solid wall influences natural convection within the enclosure in a significant way. The coupled conduction-convection energy transfer at this junction can't be neglected. One of the early pioneering studies on combined conduction-convection thermal transport in finite geometries is due to Kim and Viskanta $(1984,1985)$. They performed experimental and numerical investigations in square geometry by considering three types of thermal conditions and observed 
that the thermal variations are suppressed by thermal conductivity ratios. Later, in the same enclosure, Kaminski and Prakash (1986) made a study to predict the influence of wall thickness on buoyant flow and associated thermal transport. Numerous research works to study the influence of wall thickness on stream and thermal developments, heat energy transfers within the wall and solid-fluid interface, in various shaped enclosures Chung et al. (2001), Oztop et al. (2008), Kuznetsov and Sheremet (2011), Sheremet (2012)). In an inclined porous rectangular enclosure, Mbaye et al. (1993) examined both numerically and analytically the buoyant convection transport alongside a finite wall-thickness and conduction. Baytas et al. (2001) executed numerical analysis on buoyant convection in a conjugate square porous cavity for the arrangement consists of two conducting horizontal walls. Later, Saeid (2007) deliberated on Darcy's law to explore the conjugate convective transport in an enclosed box. Chamkha and Ismael (2013) investigated conjugate convection-conduction energy transfer set by a triangular solid wall in the square cavity under steady-state conditions. An unusual performance of thermal transfer is determined through their analysis. Recently, employing the Darcy model, Badruddin et al. (2015) scrutinized the heat transfer in a porous medium bounded by an annular region between two vertical cylinders. The impact of wall-conduction on buoyant convection of different nanofluids is also gaining attention due to many critical applications Tahmasebi et al. (2018), Zadeh et al. (2019).

A complete and thorough literature survey exhibits that the influence of the finite thickness of the thermally active wall(s) on buoyant convection has been attempted in square or triangular porous enclosed boxes. However, buoyance convection in a porous (upright) annular chamber having a finite thickness on the inner cylindrical surface has not been studied in the current literature. Hence, in the analysis, the coupled convection-conduction thermal energy transport in the porous annulus has been inspected.

\section{Mathematical modeling}

Figure 1 displays the schematic of chosen geometrical configuration. The physical geometry involves an up-right annulus chamber of breadth $\mathrm{D} \&$ elevation $H$ and is filled with liquid-saturated porous materials. The annulus chamber is made by co-axial tubular pipes with external and internal radii ro and ri, respectively. The cylindrical coordinates $(r, z)$, the velocities $(\mathrm{u}, \mathrm{w})$, and thermal settings are specified in Figure 1. The lower \& upper sections of the co-axial tubular pipes are presumed to be sealed and are adiabatic. The interior tubular surface has fixed thickness $d$ and is maintained at an elevated constant temperature, while the outer cylindrical surface is maintained at a lower uniform temperature. In addition, the subsequent suppositions are prepared to model the equations:

1. The laminar flow and invariant thermo-physical situations are presumed, except the density variations in the buoyancy term by invoking the Boussinesq approximation.

2. The fluid is Newtonian and has insignificant viscous dissipation.

3. The flow is axisymmetric.

4. The fluid properties, namely, the viscous, thermal, and solutal diffusivities represented. 
5. The viscous and ohmic dissipations, radiation effects, and compressibility are neglected.

6. The porous medium is presumed to be rigid, isotropic \& homogeneous.

7. The exact temperature is assumed at fluid \& solid-phase everywhere, and the Local Thermal Equilibrium assumption is applied.

8. The Brinkman-extended Darcy model is chosen, and as a result, the term with Forchheimer quadratic drag in the momentum equation is ignored.

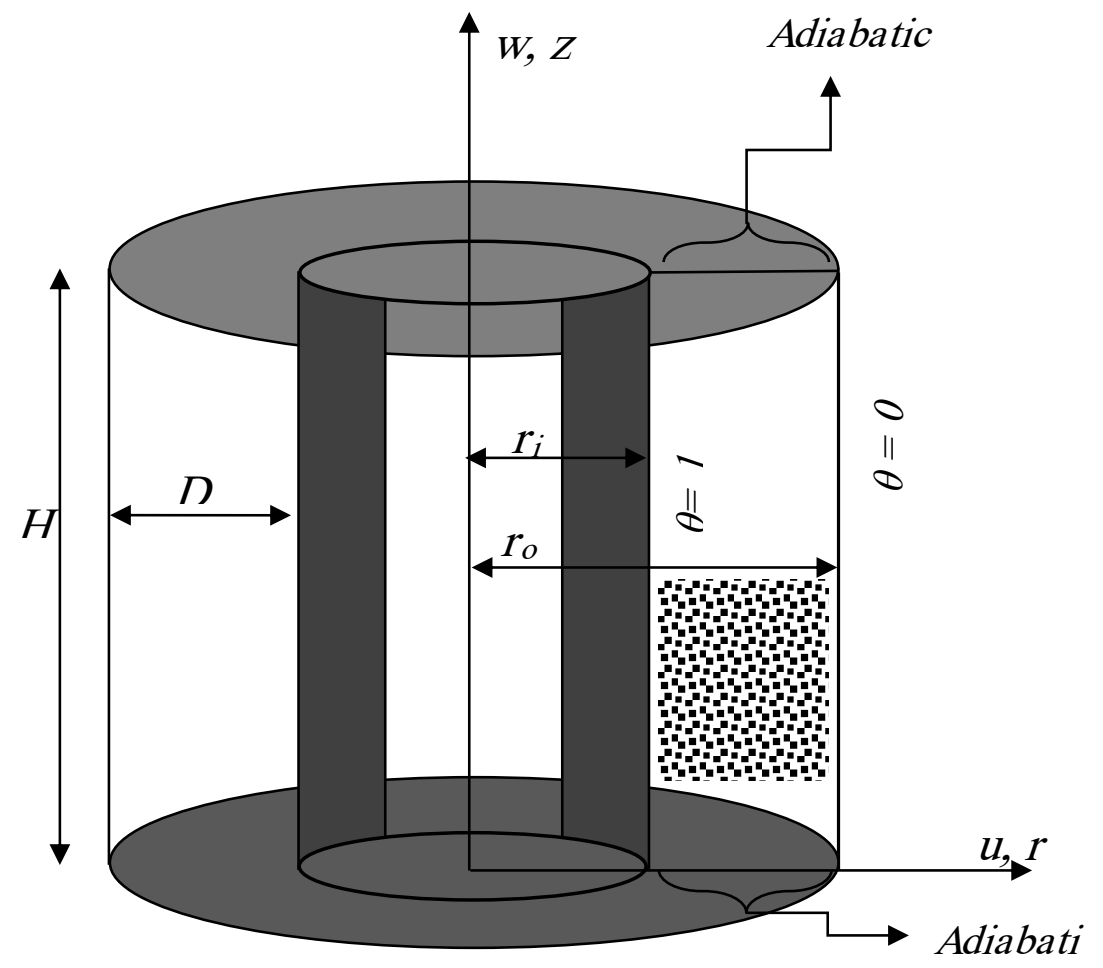

Fig. 1. Physical configuration, coordinate system, and boundary conditions.

By applying the above suppositions, the equations model the conservation of mass, momentum and energy are:

$$
\begin{gathered}
\nabla \cdot \vec{q}=0 \\
\frac{1}{\phi} \frac{\partial \vec{q}}{\partial t^{*}}+\frac{1}{\phi^{2}}(\vec{q} \cdot \nabla) \vec{q}=-\frac{1}{\rho_{0}} \nabla p+\frac{v}{\phi}\left[\nabla_{1}^{2} \vec{q}\right]-\frac{v}{K} \vec{q}+\vec{g} \beta\left(\theta-\theta_{h}\right)
\end{gathered}
$$

For fluid region, the energy equation is

$$
\frac{\partial \theta_{f}}{\partial t^{*}}+(\stackrel{\mathrm{r}}{q} \cdot \nabla) \theta_{f}=\alpha_{f}\left[\nabla_{1}^{2} \theta_{f}\right]
$$

For solid portion, the energy equation is

$$
\frac{\partial \theta_{w}}{\partial t^{*}}=\alpha_{w}\left[\nabla_{1}^{2} \theta_{w}\right]
$$


Where $\stackrel{1}{q}=(u, w)$ and $\nabla_{1}^{2}=\frac{\partial^{2}}{\partial r^{2}}+\frac{1}{r} \frac{\partial}{\partial r}+\frac{\partial^{2}}{\partial z^{2}}$.

The following transformations are considered in this study for non-dimensional process:

$$
\begin{aligned}
& (R, Z)=(r, z) / D, \quad(U, W)=(u, w) /\left(\alpha_{f} / D\right), t=t^{*} /\left(D^{2} / \alpha_{f}\right), T=\left(\theta-\theta_{h}\right) / \Delta \theta, \\
& P=p /\left(\rho_{0} \alpha_{f}^{2} / D^{2}\right), \quad \zeta=\zeta * /\left(\alpha_{f} / D^{2}\right), \Psi=\psi /\left(D \alpha_{f}\right) \text {, where } D=r_{o}-r_{i}, \Delta \theta=\theta_{h}-\theta_{c}
\end{aligned}
$$

The resulting non dimensional governing equations after eliminating the pressure term, using cross differentiation, from the equations (2) are

$$
\begin{gathered}
\frac{\partial T_{w}}{\partial t}=\frac{\alpha_{w}}{\alpha_{f}}\left[\frac{\partial^{2} T_{w}}{\partial R^{2}}+\frac{1}{R} \frac{\partial T_{w}}{\partial R}+\frac{\partial^{2} T_{w}}{\partial Z^{2}}\right] \\
\frac{\partial T_{f}}{\partial t}+U \frac{\partial T_{f}}{\partial R}+W \frac{\partial T_{f}}{\partial Z}=\left[\frac{\partial^{2} T_{f}}{\partial R^{2}}+\frac{1}{R} \frac{\partial T_{f}}{\partial R}+\frac{\partial^{2} T_{f}}{\partial Z^{2}}\right] \\
\frac{1}{\phi} \frac{\partial \zeta}{\partial t}+\frac{C_{1}}{\phi^{2}}\left[U \frac{\partial \zeta}{\partial R}+W \frac{\partial \zeta}{\partial Z}-\frac{U \zeta}{R}\right]=\frac{C_{2} \operatorname{Pr}}{\phi}\left[\frac{\partial^{2} \zeta}{\partial R^{2}}+\frac{1}{R} \frac{\partial \zeta}{\partial R}+\frac{\partial^{2} \zeta}{\partial Z^{2}}-\frac{\zeta}{R^{2}}\right] \\
\frac{\partial^{2} \Psi}{\partial R^{2}}-\frac{1}{R} \frac{\partial \Psi}{\partial R}+\frac{\partial^{2} \Psi}{\partial Z^{2}}=R \zeta \\
U R=\frac{\partial \Psi}{\partial Z}, \quad W R=-\frac{\partial T}{\partial R}
\end{gathered}
$$

It is to be noted that the constants $\mathrm{C}_{1}$ and $\mathrm{C}_{2}$ in Equation (7) can be assigned the values 0 or 1 to generate two different porous media models Nithiarasu et al. (1997). For $\mathrm{C} 1=\mathrm{C} 2=0$, the vorticity form of momentum Eq. (7) reduces to Darcy model, whereas for $\mathrm{C} 1=\mathrm{C} 2=1$, the Equation (7) represents the Brinkman-extended Darcy model.

The dimensionless initial and boundary conditions are:

At $t=0$, the fluid is motionless, and temperature is uniform everywhere

At $t=0$, thermal conditions at the inner and outer cylinders are 1 and 0 respectively, upper, and lower walls are kept adiabatic, i.e.

$$
\frac{\partial T_{w}}{\partial \eta}=\frac{\partial T_{f}}{\partial \eta}=0
$$

At the solid-fluid interface, the below condition is employed.

$$
T_{f}=T_{w} \text { and } \frac{\partial T_{f}}{\partial R}=K_{r} \frac{\partial T_{w}}{\partial R} \text {. }
$$


The following seven dimensionless parameters arise in the present study (three geometrical and four physical parameters):

$$
\lambda=\frac{r_{0}}{r_{i}} \text { radius ratio, } A=\frac{H}{D} \text { aspect ratio, }
$$

$\varepsilon=\frac{d}{D}$ dimensionless wall thickness,

$K_{r}=\frac{k_{w}}{k_{f}}$ thermal conductivity ratio, $\operatorname{Pr}=\frac{v}{\alpha}$ Prandtl number, $D a=\frac{K}{D^{2}}$ Darcy number, and $R a=\frac{g \beta\left(\theta_{h}-\theta_{c}\right) D^{3}}{v \alpha}$ Rayleigh number.

The wall vorticity can be calculated using Taylor's series expansion of wall stream function values and interior values of $\psi$. In this paper, the boundary vorticities are computed from the relation: $\zeta_{b}=\frac{8 \psi_{b+1}-\psi_{b+2}}{2(\Delta \eta)^{2}}$, where $b$ indicates the value of $\zeta$ at boundary and $\Delta \eta$ is the spacing between two grid points. In heat transport analysis, the design engineer is interested in investigating the qualitative and quantitative information on the chosen problem. After obtaining the steady-state solutions from the governing equations (6) - (9), the parameter of quantitative interest is thermal transport rate and is valued from local $(N u)$ and total $(\overline{N u})$ Nusselt numbers. The local $N u$ at the surface is measured from $N u=-\frac{\partial T}{\partial R}$. The global energy transport rate is computed from the averaged Nusselt number and is calculated at the inner wall $\overline{N u_{W}}$ and interface $\overline{N u_{I}}$ from below formulae:

$$
\overline{N u_{W}}=\frac{1}{A} \int_{0}^{A} N u_{W} d z \quad \text { and } \quad \overline{N u_{I}}=\frac{1}{A} \int_{0}^{A} N u_{I} d z
$$

\section{Numerical Technique and Justification}

To perform a detailed analysis of the chosen problem, the physical laws are transformed to a system of partial differential equations (PDE). Since the model equations are coupled and nonlinear, analytical solutions to these equations are not possible. Hence, by utilizing the available high-speed computing resources, the governing "partial differential equations" are solved numerically using finite difference techniques, namely the Successive Line Over Relaxation (SLOR) \& Alternating Direction Implicit (ADI) methods. In particular, transient PDEs are discretized by the ADI method. The energy equation without advection terms is applied in the solid section, whereas the typical energy equation is involved in the fluid section. However, the vorticity-stream function relation is discretized by the SLOR method with the proper choice of relaxation parameter. The global $N u$ is estimated by the numerical integration. Further, to solve the finite difference equations arising from the discretization process, an in-house code is written, 
and our results are compared with the existing standard benchmark predictions before the present simulations. The iteration process employed to find the stream function, temperature, and vorticity fields is repeated until the following convergence criterion is satisfied: $\max \left[\frac{\left|\chi_{i, j}^{n+1}-\chi_{i, j}^{n}\right|}{\left|\chi_{i, j}^{n+1}\right|}\right] \leq \delta$

The above condition $\chi$ stands for $\Psi$ or T, where $i, j$ denote the grid locations, $n$ symbolizes the time iteration and $\delta$ is the convergence norm. The detailed information on numerical method is provided in our earlier works; Sankar and Do (2010), Sankar et al. (2011), Sankar et al. (2013) and are not repeated here for brevity.

\subsection{Grid sensitivity analysis and Validation}

All simulations are thoroughly tested for the independence of grid sizes by generating uniform grids in the annular domain. To check grid independency, the averaged Nusselt numbers $(\overline{N u})$ are obtained by employing different grid sizes of $51 \times 51,81 \times 81,101 \times 101$, and $121 \times 121$. After careful observation of $\overline{N u}$ each of the chosen grid sizes, an optimum grid size is selected at which the averaged Nusselt number $\overline{N u}$ is not varied significantly with a further increment in grids. Also, the utmost variation between $101 \times 101$ and $121 \times 121$ grids is found within $0.1 \%$, and hence a $101 \times 101$ grid is used in all calculations. A code using ForTran is developed for solving the algebraic equations and verified with the earlier results.

Table 1: Averaged Nusselt number with the results of Prasad (1986) at $A=1$ (Darcy model).

\begin{tabular}{|c|c|c|c|c|}
\hline Radius ratio $(\lambda)$ & $\begin{array}{l}\text { Darcy-Rayleigh } \\
\text { number }\left(\mathrm{Ra}_{\mathrm{D}}\right)\end{array}$ & $\begin{array}{l}\text { Prasad } \\
(1986)\end{array}$ & Present study & $\begin{array}{l}\text { Relative } \\
\text { difference (\%) }\end{array}$ \\
\hline \multirow[t]{2}{*}{2} & $10^{3}$ & 6.4934 & 6.4815 & 0.18 \\
\hline & $10^{4}$ & 16.0498 & 16.0271 & 0.14 \\
\hline \multirow[t]{2}{*}{3} & $10^{3}$ & 7.1659 & 7.1804 & 0.20 \\
\hline & $10^{4}$ & 17.2691 & 17.2226 & 0.27 \\
\hline \multirow[t]{2}{*}{5} & $10^{3}$ & 8.0036 & 8.0262 & 0.28 \\
\hline & $10^{4}$ & 18.8055 & 18.8631 & 0.31 \\
\hline \multirow[t]{2}{*}{10} & $10^{3}$ & 9.3975 & 9.4452 & 0.51 \\
\hline & $10^{4}$ & 20.7498 & 20.8325 & 0.40 \\
\hline
\end{tabular}

Initially, the current results are corroborated with different benchmark results for the limiting cases. First, for zero wall thickness, the simulations are performed in a differently heated porous annular geometry. Figure 2 depicts the comparison of isotherm and streamlines contours estimated from our simulations and those of Shivakumara et al. (1995). The comparison reveals good agreement with the porous annular enclosure for zero wall thickness. Finally, for the unit radius ratio case $(\lambda=1)$, the present problem reduces to the square enclosure. $\lambda=1$, The global Nusselt numbers are determined from current simulations for the Darcy model and compared with Prasad 
(1984) in a porous annulus. Table 1 reveals an excellent agreement with the average Nusselt numbers measured from Prasad (1984) in the annular cavity without a wall thickness.

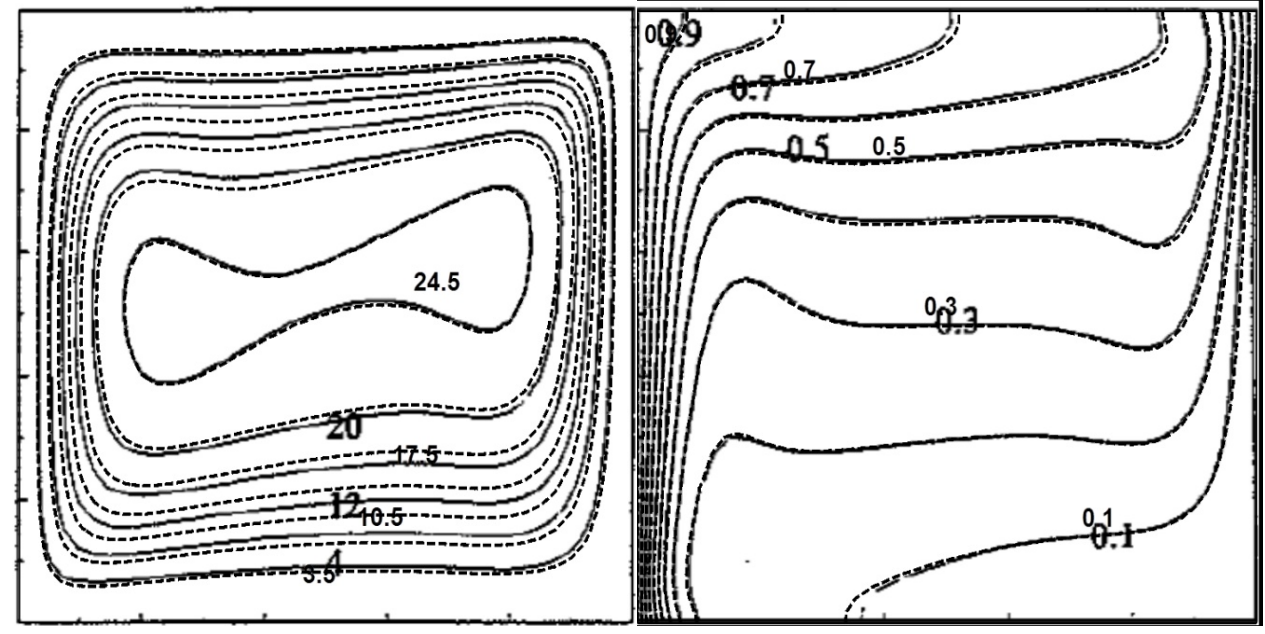

Fig. 2. Comparison of streamlines and isotherms between the present results and that of Shivakumara et al. (1995) for $\mathrm{Ra}=10^{6}, \mathrm{Da}=10^{-2}, \Lambda=3, \lambda=2, \mathrm{~A}=1$ and $\operatorname{Pr}=7.0$. Discontinuous lines correspond to present results, and continuous lines correspond to Shivakumara et al. (1995).

\section{Results and Discussion}

In this division, the influences of various geometric and physical parameters on conjugate convective stream and thermal transport have been discussed in detail. More importance is given to the cases involving wall-thickness and conductivity ratio amongst the rigid wall and fluid. The results are offered in terms of isotherms and streamlines to interpret the temperature distribution and the circulation of the fluid, respectively. Apart from this, a more detailed discussion has been performed using the plots of local and averaged Nusselt numbers. In the present analysis, four different wall-thickness ( $\varepsilon=0.1,0.2,0.3$ and 0.5 ) and three conductivity ratios $\left(K_{r}=0.5,5.0\right.$ and 10.0) are considered. Further, the vast choice of physical parameters, namely Rayleigh (Ra) and Darcy (Da) numbers $\left(10^{3} \leq \mathrm{Ra} \leq 10^{6}\right.$ and $\left.10^{-5} \leq \mathrm{Da} \leq 10^{-1}\right)$, are considered for the analysis. However, the respective values of aspect and radius ratios, Prandtl number are fixed at $\mathrm{A}=1, \lambda=2$ and $\operatorname{Pr}=$ 0.707 .

\subsection{Influence of $\mathrm{Da}$, conductivity ratio, and wall thickness on streamlines and isotherms}

Figure 3 exhibits the impact of wall conductivity ratio $\left(K_{r}\right)$ on the isotherms and streamlines for $\mathrm{Da}=10^{-5}, \varepsilon=0.2$, and $\mathrm{Ra}=10^{3}$. The streamlines are displayed to the right, and the isotherms are shown to the left side of the figure. The streamline reveals a larger circular rotating cell that occupies the entire region, the circulation of streamlines is in a clockwise direction as indicated by the negative sign. The circulation power of the streamlines rises with a growth in the wall conductivity ratio $K_{r}$. This is because the fluid closer to the solid interface wall (annulus inner 
wall) receives the heat from the wall and starts moving towards the cold outer wall due to the difference in the temperature. This phenomenon gives rise to a single clockwise rotating cell within the porous annular region. The isotherms displayed on the left of Figure 3 appear to be more vertical, which shows that the thermal energy transport in the porous annulus is by conduction. This can be described as the high resistivity offered by the wall to the porous medium. As the thermal conductivity ratio rises, the solid fence becomes conductive to penetrate the heat to the porous region, which leads to the convection kind of heat energy transference in the porous area. Again, as the porosity decreases from $\mathrm{Da}=10^{-5}$ to $\mathrm{Da}=10^{-3}$, the strength of the circulation becomes stronger (see Figure 4), and the isotherms in the porous area become horizontal, which implies that the convection is dominating the flow.
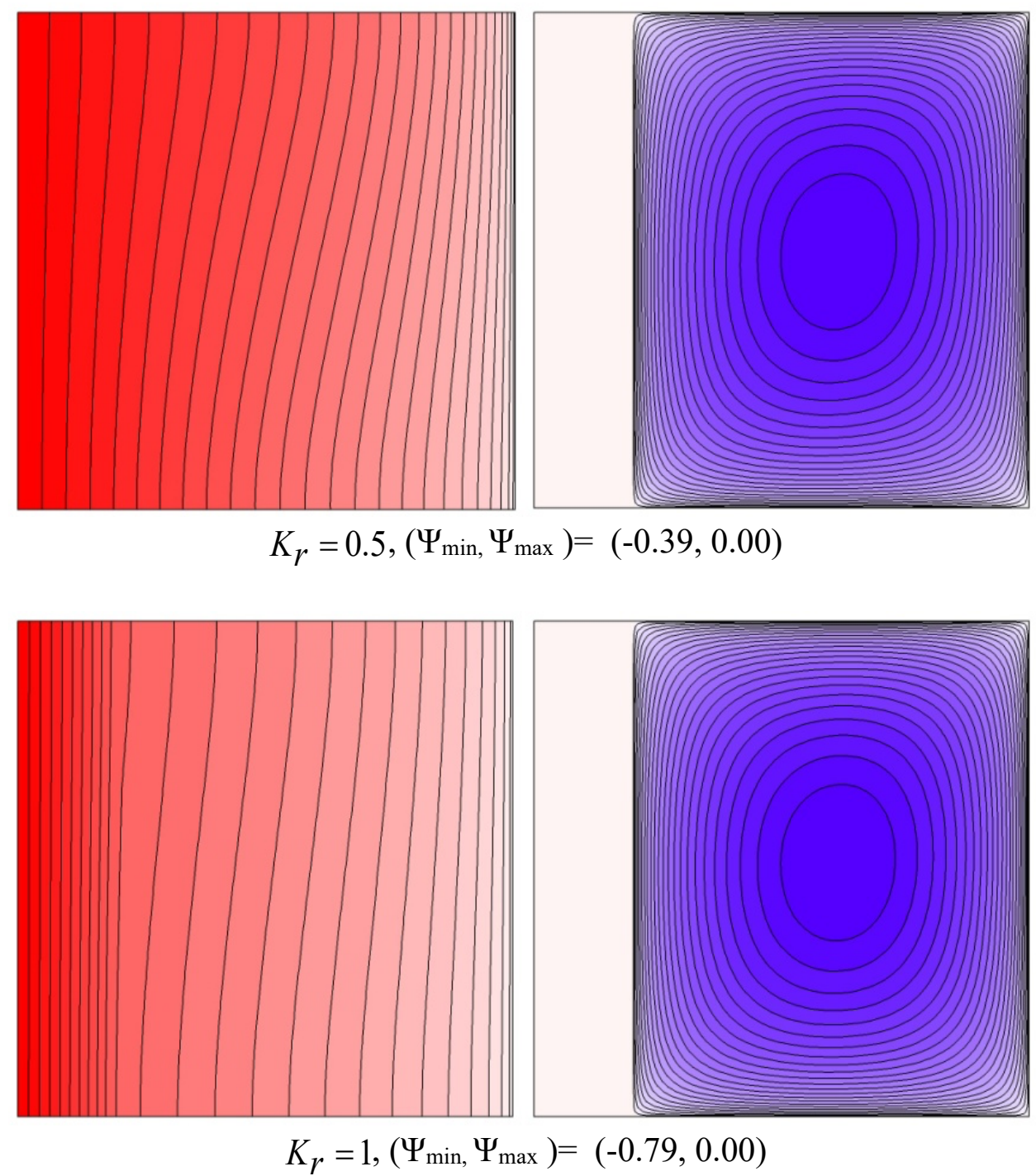


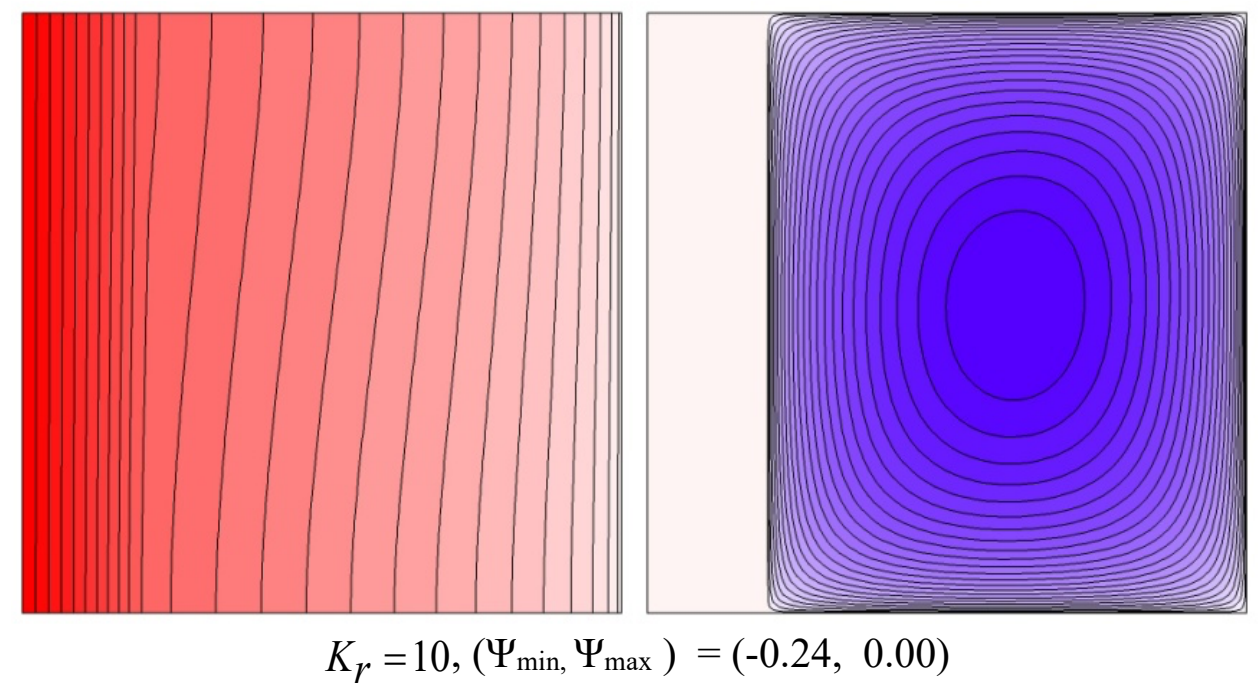

Fig. 3. Effect of $K_{r}$ on isotherms and streamlines for $\mathrm{Da}=10^{-5}, \varepsilon=0.2$ and $\mathrm{Ra}=10^{6}$.
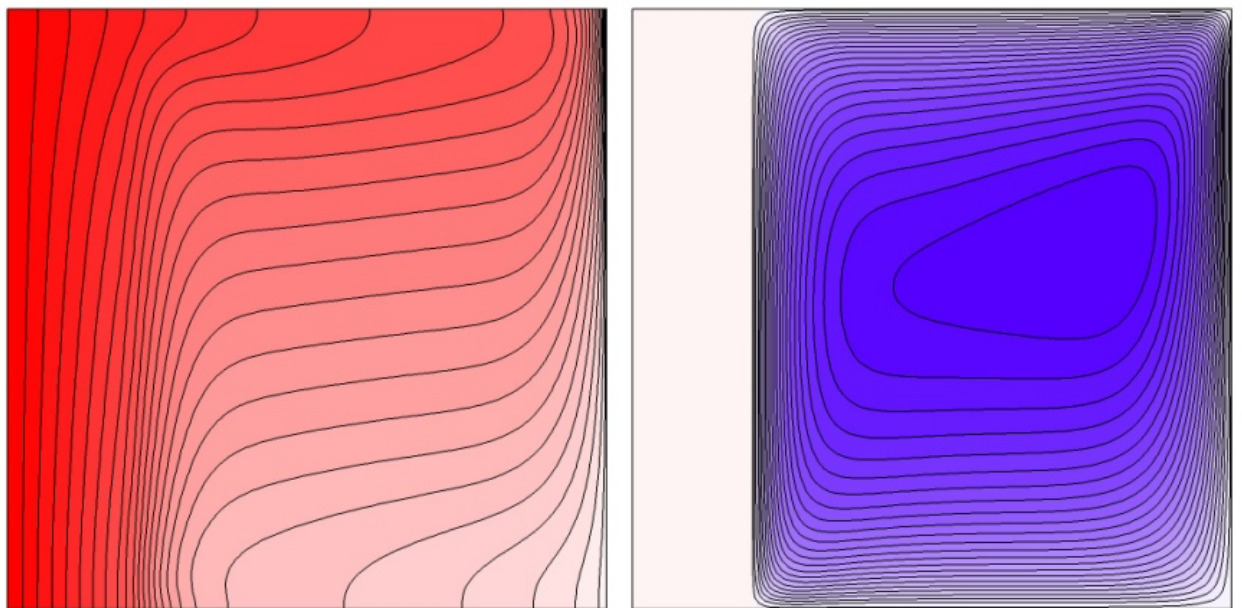

$$
K_{r}=0.5,\left(\Psi_{\min }, \Psi_{\max }\right)=(-4.12,0.00)
$$
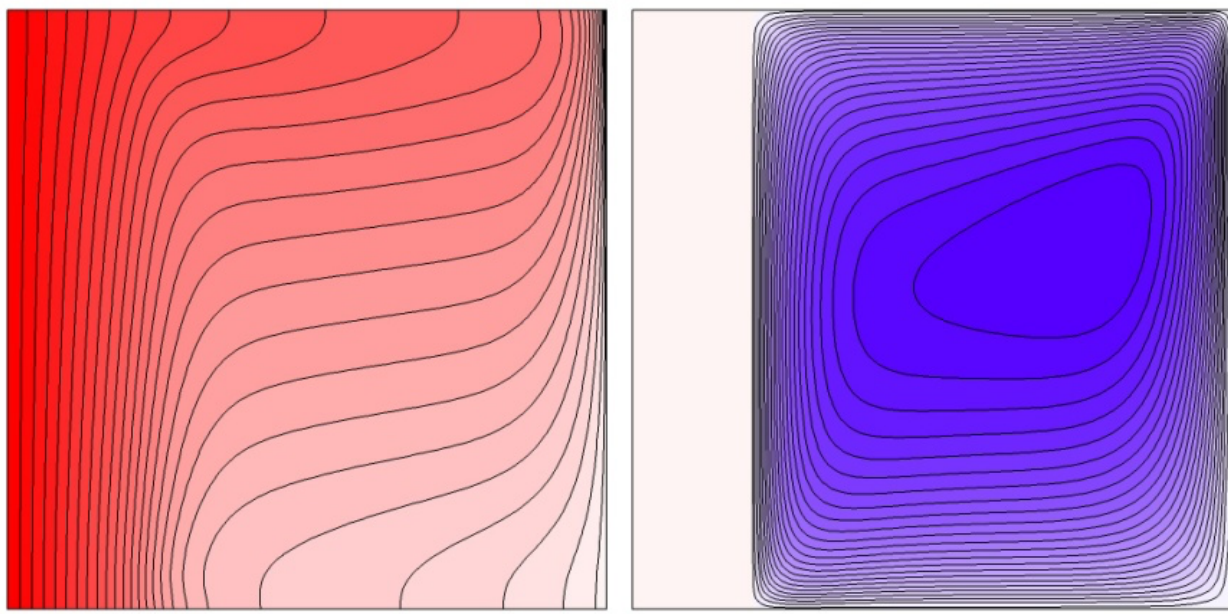

$$
K_{r}=1,\left(\Psi_{\min }, \Psi_{\max }\right)=(-12.15,0.00)
$$



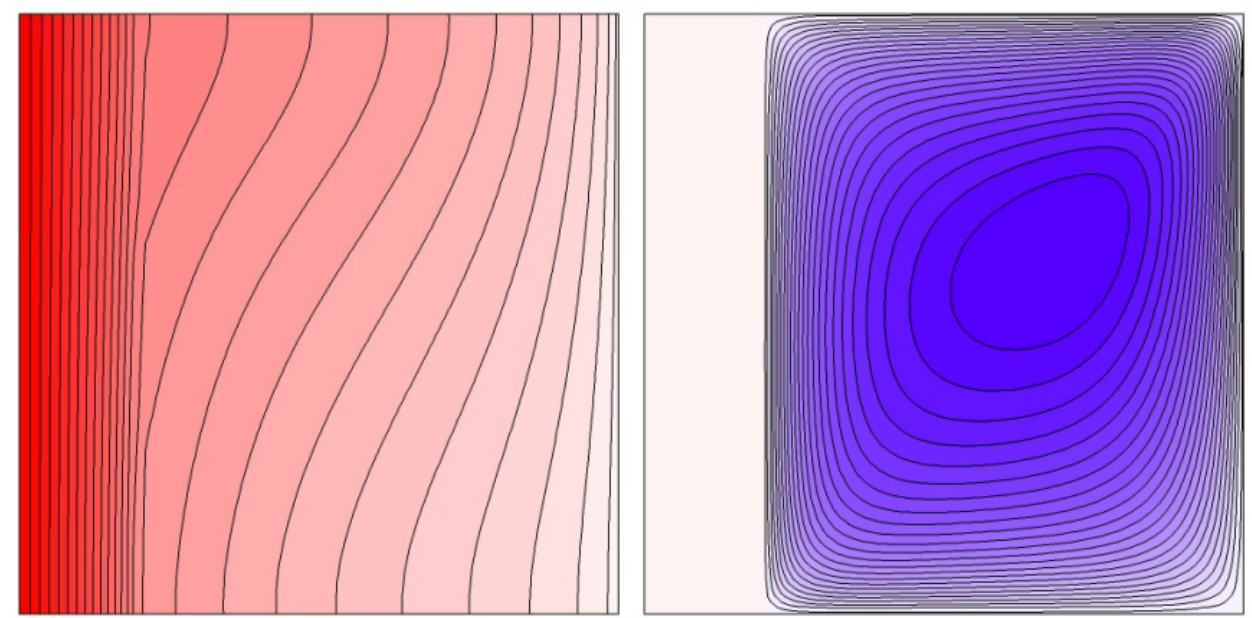

$$
K_{r}=10,\left(\Psi_{\min ,} \Psi_{\max }\right)=(-6.00,0.00)
$$

Fig. 4. Effect of $K_{r}$ on isotherms and streamlines for $\mathrm{Da}=10^{-3}, \varepsilon=0.2$ and $\mathrm{Ra}=10^{6}$.
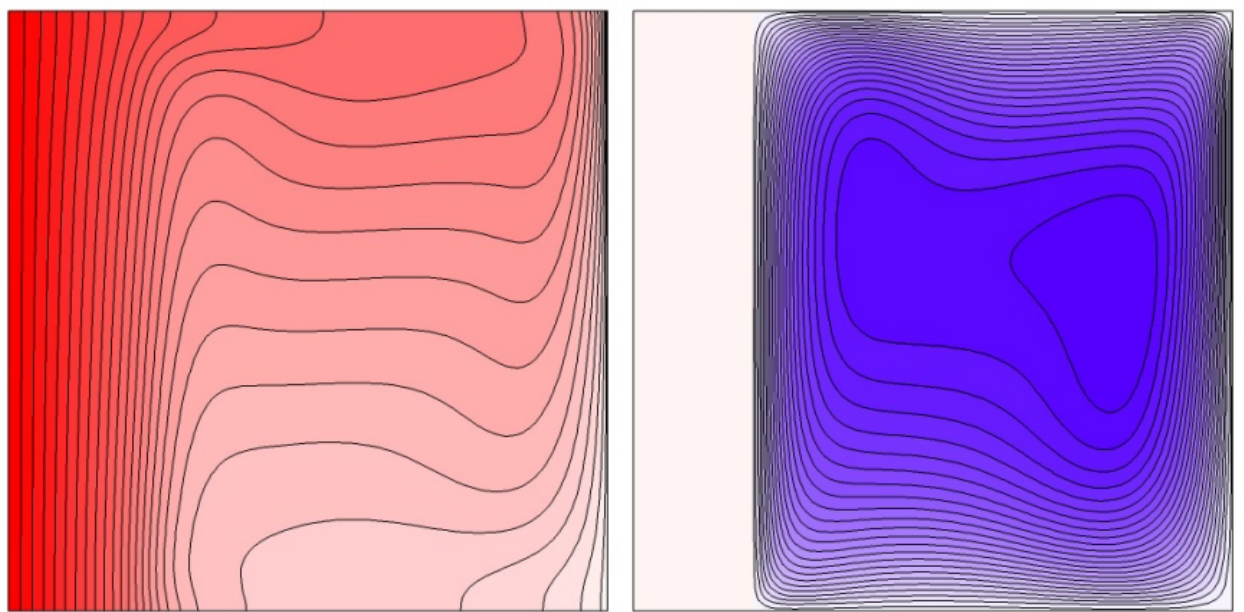

$$
K_{r}=0.5,\left(\Psi_{\min }, \Psi_{\max }\right)=(-14.23,0.00)
$$
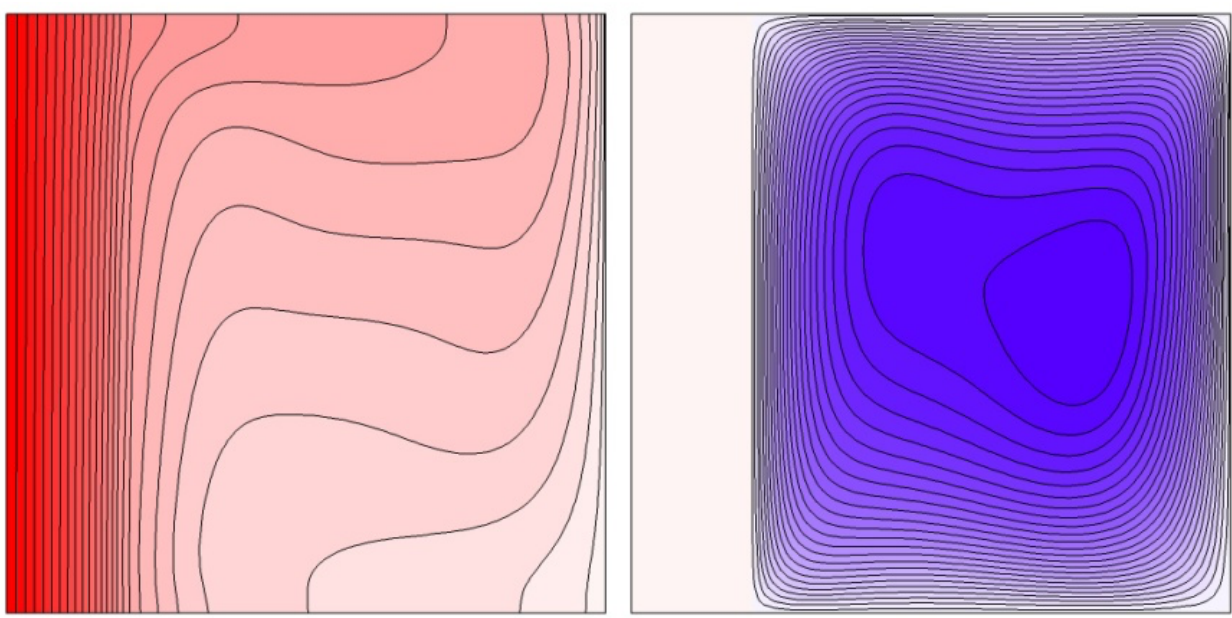

$$
K_{r}=1,\left(\Psi_{\min }, \Psi_{\max }\right)=(-18.67,0.00)
$$



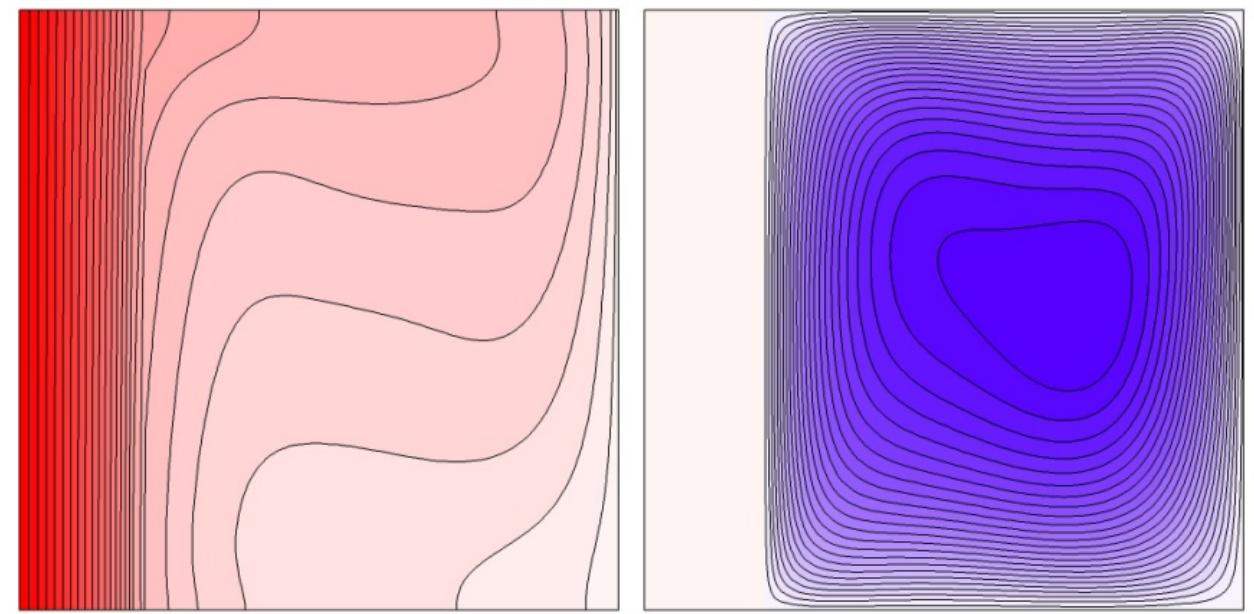

$$
K_{r}=10,\left(\Psi_{\min }, \Psi_{\max }\right)=(-12.49,0.00)
$$

Fig. 5. Effect of $K_{r}$ on isotherms and streamlines for $\mathrm{Da}=10^{-1}, \varepsilon=0.2$ and $\mathrm{Ra}=10^{6}$.
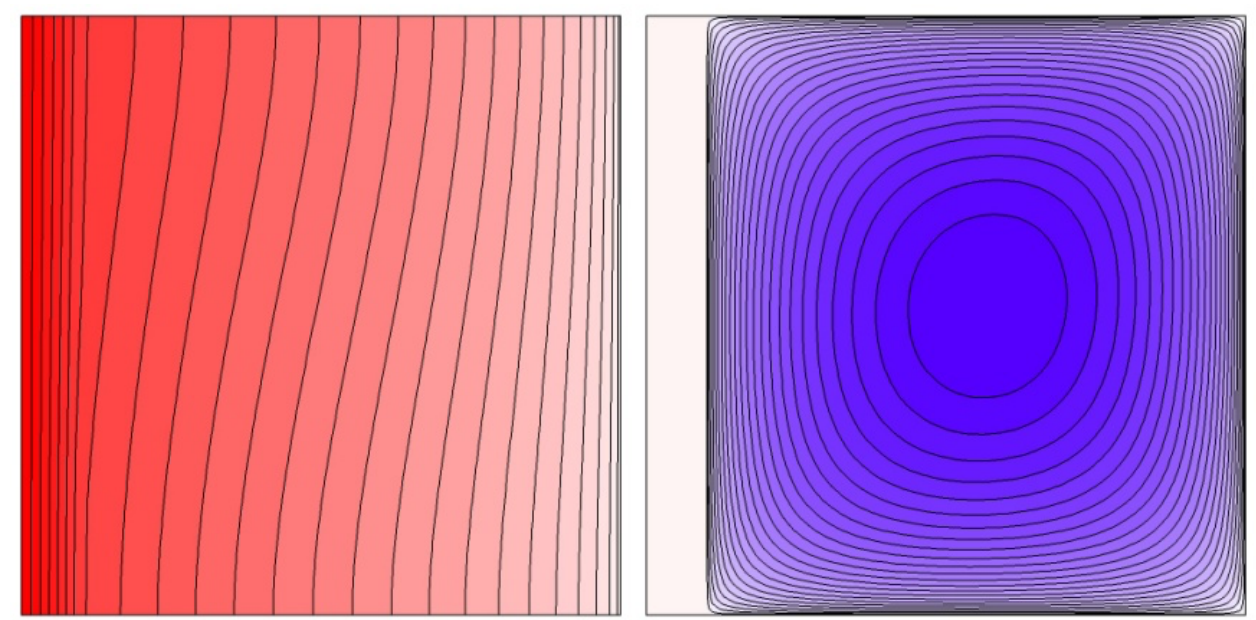

$$
\boldsymbol{\varepsilon}=0.1,\left(\Psi_{\min }, \Psi_{\max }\right)=(-0.59,0.00)
$$

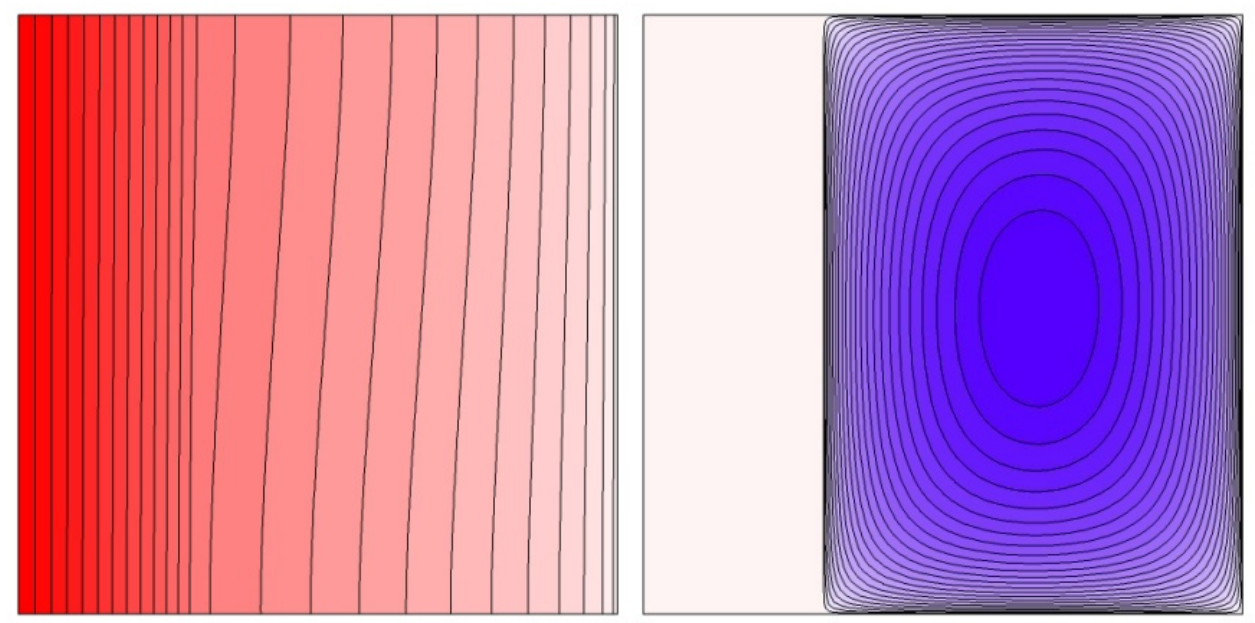

$$
\boldsymbol{\varepsilon}=0.3,\left(\Psi_{\min }, \Psi_{\max }\right)=(-0.27,0.00)
$$



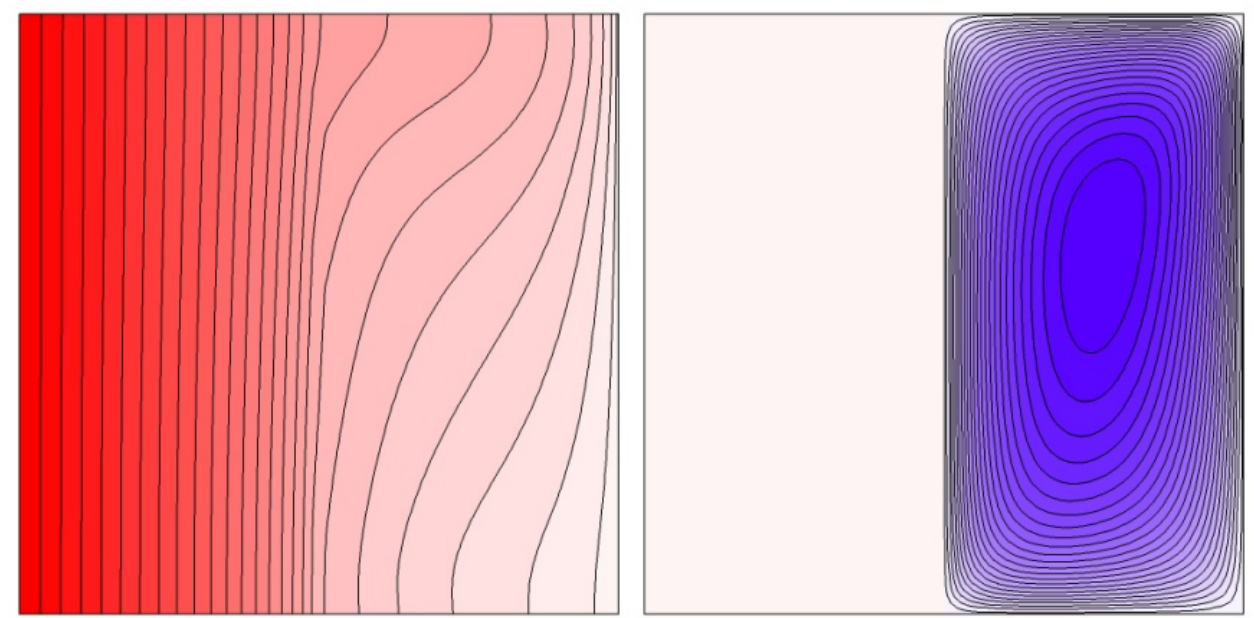

$$
\boldsymbol{\varepsilon}=0.5,\left(\Psi_{\min }, \Psi_{\max }\right)=(-4.99,0.00)
$$

Fig. 6. Effect of $\varepsilon$ on isotherms and streamlines for $\mathrm{Da}=10^{-5}, K_{r}=5$ and $\mathrm{Ra}=10^{6}$.
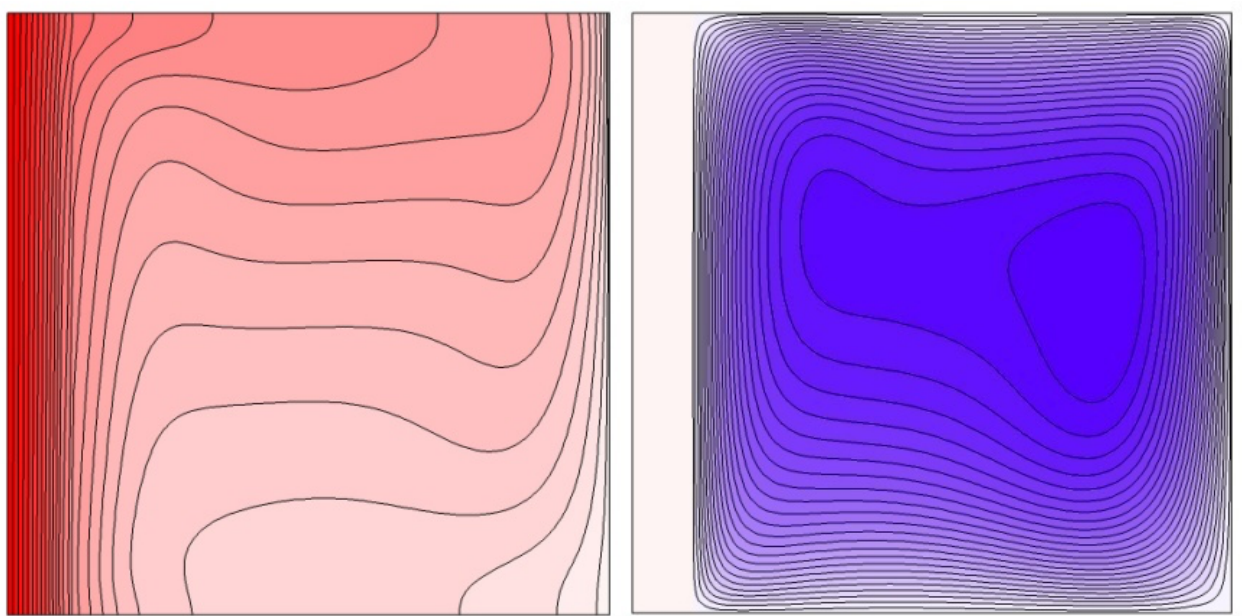

$$
\boldsymbol{\varepsilon}=0.1,\left(\Psi_{\min }, \Psi_{\max }\right)=(-15.95,0.0)
$$

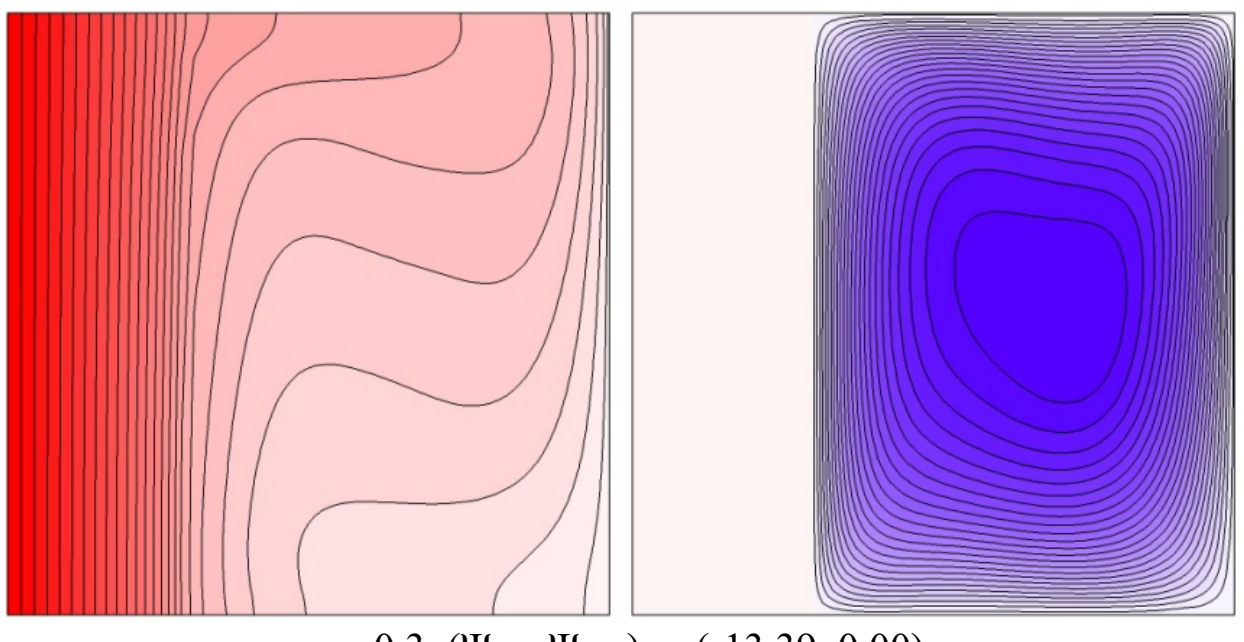

$$
\boldsymbol{\varepsilon}=0.3,\left(\Psi_{\min }, \Psi_{\max }\right)=(-13.39,0.00)
$$




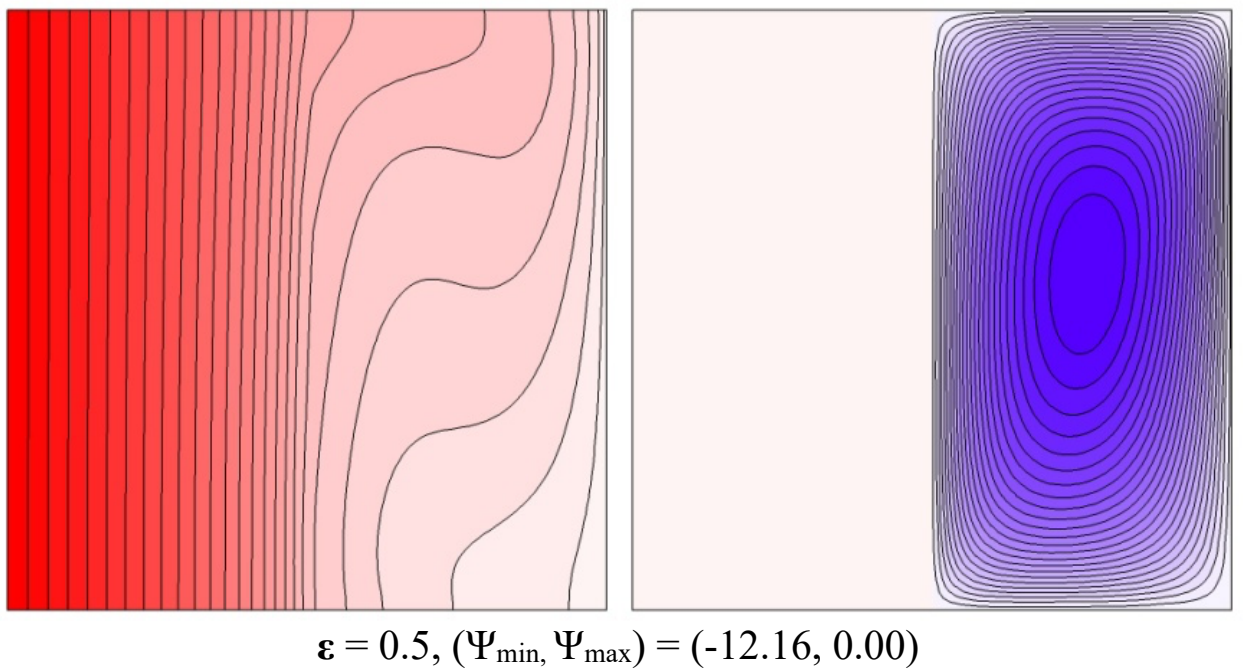

Fig. 7. Effect of $\varepsilon$ on isotherms and streamlines for $\mathrm{Da}=10^{-1}, K_{r}=5$ and $\mathrm{Ra}=10^{6}$.

As the value $K_{r}$ is increased from 0.5 to 10 , the isotherms of the $\mathrm{Da}=10^{-3}$ form a vertical pattern in the porous domain. This can be elucidated as the resistivity of the heat energy transfer is more by the porosity of the fluid than the solid wall conductivity ratio. Again by decreasing the porosity to $\mathrm{Da}=10^{-1}$ (see Figure 5), it can be observed that isotherms in the porous domain become more horizontal for an increase in $K_{r}$, which gives clear evidence of heat transfer taking place through natural convection. Figures 6 and 7 show the isotherms and flow field for different values of wall thickness for two diverse magnitudes of Darcy number $\left(\mathrm{Da}=10^{-1} \& \mathrm{Da}=10^{-5}\right)$. It can be detected that by increasing the wall thickness, the thermal gradient across the porous part decreases for both the cases of Darcy number. This is because most heat transference is restricted through the solid wall. It can be perceived from Figure 6 that for a thin wall $\varepsilon=0.1$, the isotherms are vertical throughout the concrete wall and the porous domain for $\mathrm{Da}=10^{-5}$. However, for the same wall thickness and $\mathrm{Da}=10^{-1}$, the isotherms in Figure 7 appear to be vertical in the solid region and horizontal in the porous domain. The streamlines gain more strength showing that the convection type of heat transfer dominates the porous domain. This phenomenon can be observed for all the wall thickness for both the values of Darcy numbers. Therefore, it is found that declining the wall thickness and the porosity of the fluid increases the heat transference in the porous domain. 


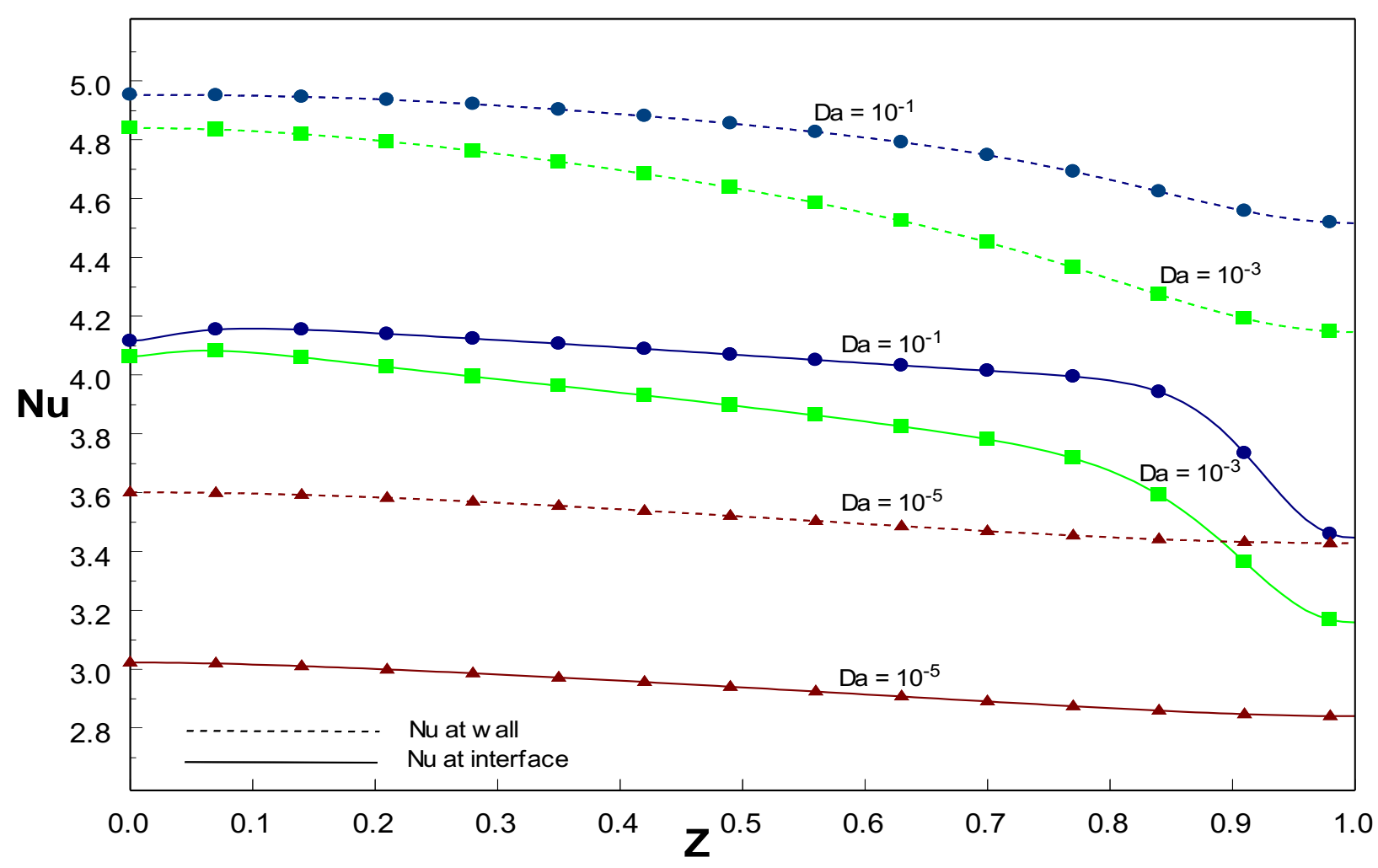

Fig. 8. Effect of Darcy number on the local Nusselt number for $\varepsilon=0.2, K_{r}=5$ and $\mathrm{Ra}=10^{6}$.

\subsection{Impact of $\mathrm{Kr}, \mathrm{Da}$, and $\varepsilon$ on the local Nusselt number}

The deviation of thermal flux along with the fluid-wall interface and the solid wall for different $\mathrm{Da}$ and constant solid wall thickness $\varepsilon=0.2$, conductivity ratio $K_{r}=5$, and $\mathrm{Ra}=10^{6}$ is shown in Fig. 8. A comparison is made for thermal flux along the concrete wall and the wall/fluid interface in the $\mathrm{Z}$ direction, the dotted curve represents the local Nusselt number alongside a solid wall, and the solid curve represents the local $N u$ along with the fluid-wall interface. It is detected that the $N u$ along the border is always higher than that of the interface. This is expected because the restriction of heat transfers to the porous domain by the wall thickness $\varepsilon$. Also, it can be detected that for lower Da values, the lower $N u$ is recorded. As the value of Da rises, heat transfer along the wall and the interface also increases, but a slight decrease at the top of the wall is noticed due to the corner effect. Figure 9 depicts the deviation of local $\mathrm{Nu}$ along the wall and the fluid-wall interface for several wall thicknesses and for fixed $K_{r}=5, \mathrm{Da}=10^{-3}$ and $\mathrm{Ra}=10^{6}$. In this case, it is also perceived that reducing the wall thickness increases the local $N u$ and for growing the wall thickness reduces the heat transfer. A similar explanation can be given for the wall conductivity ratio as exemplified in Figure 10 as the conductivity ratio rises, the heat flux also increases. This means high conductive walls offer low resistance to the flow of heat. 


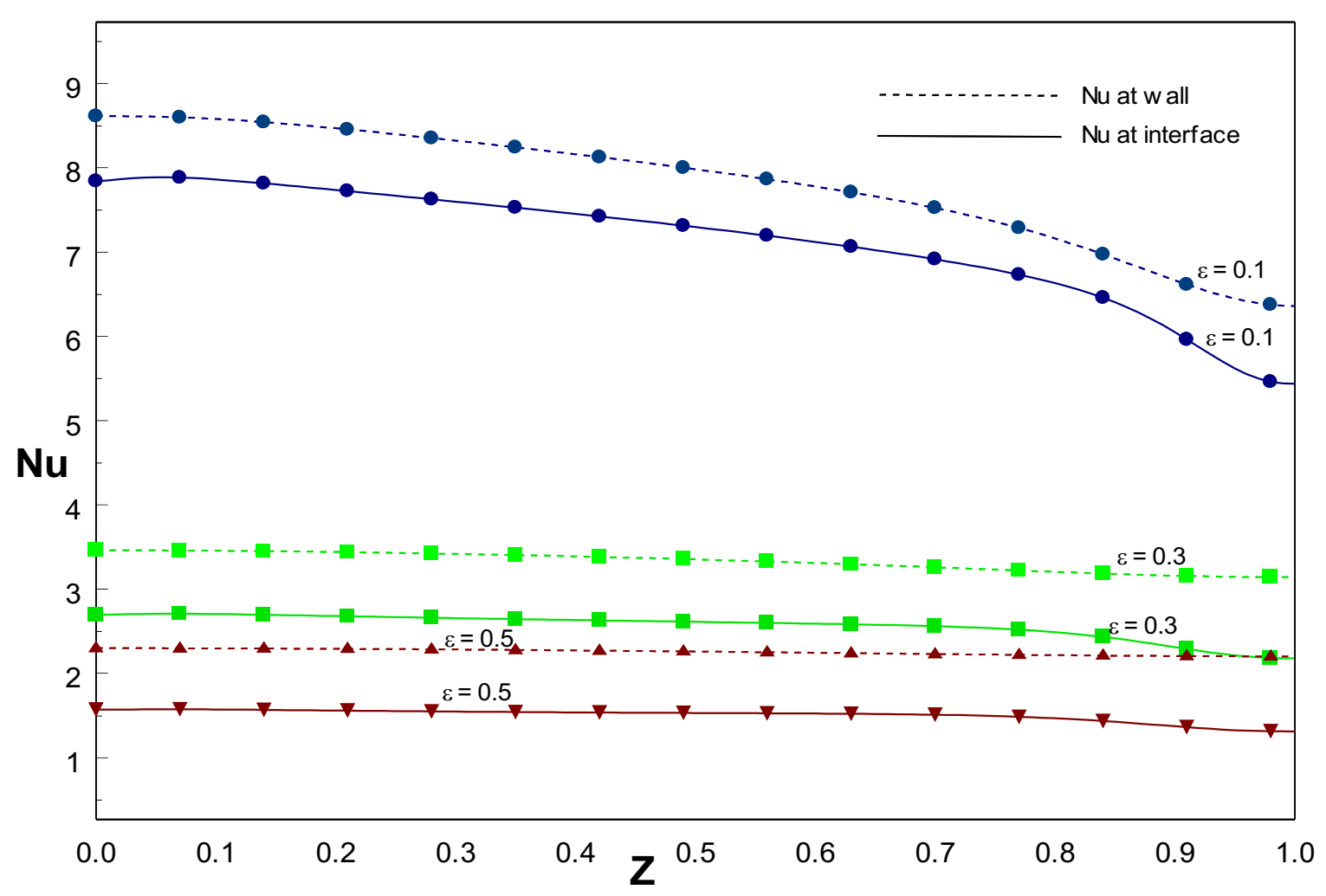

Fig. 9. Effect of $\varepsilon$ on the local Nusselt number for $\mathrm{Ra}=10^{6}, \mathrm{Da}=10^{-3}$ and $K_{r}=5$.

4.3 Impact of $\mathrm{Da}, \mathrm{Kr}$, and $\varepsilon$ on the averaged Nusselt number.

The influence of wall thickness on the averaged Nusselt number with varying Darcy numbers is plotted in Figure 11. It is detected that for thicker walls $(\varepsilon=0.5$ and $\varepsilon=0.3)$, heat transport across the cylinder surface to the interface is very minimum for increasing the values of Darcy number. However, for lesser wall thickness $(\varepsilon=0.1)$ leads to a rise in heat energy transfer with an increase of Darcy number, and the highest average $\mathrm{Nu}$ is reached for this wall thickness. Figure 12 demonstrates the outcome of conductivity ratio on the averaged Nusselt number for several Darcy numbers. As discoursed earlier, the heat transmission rises with a rise in the wall conductivity ratio. Here also it can be observed that the averaged $N u$ increases for rising the value of $D a$ for a constant wall thickness. In general, the average $\mathrm{Nu}$ increases as $\mathrm{Da}$ increases for all the conductivity ratios up to $\mathrm{Da}=10^{-3}$, a further rise in $\mathrm{Da}$ it is seen that the averaged Nusselt number remains constant for $10^{-3} \leq \mathrm{Da} \leq 10^{-1}$. The influence of Rayleigh number on the averaged Nusselt number for diverse magnitudes of $D a$ is illustrated in Figure 13. It can be observed that the averaged $\mathrm{Nu}$ remains constant for the rising Rayleigh number up to $10^{6}$ for the Darcy number Da $=10^{-5}$. As the Rayleigh number is elevated or the porosity decreases, the averaged Nusselt number rises. 


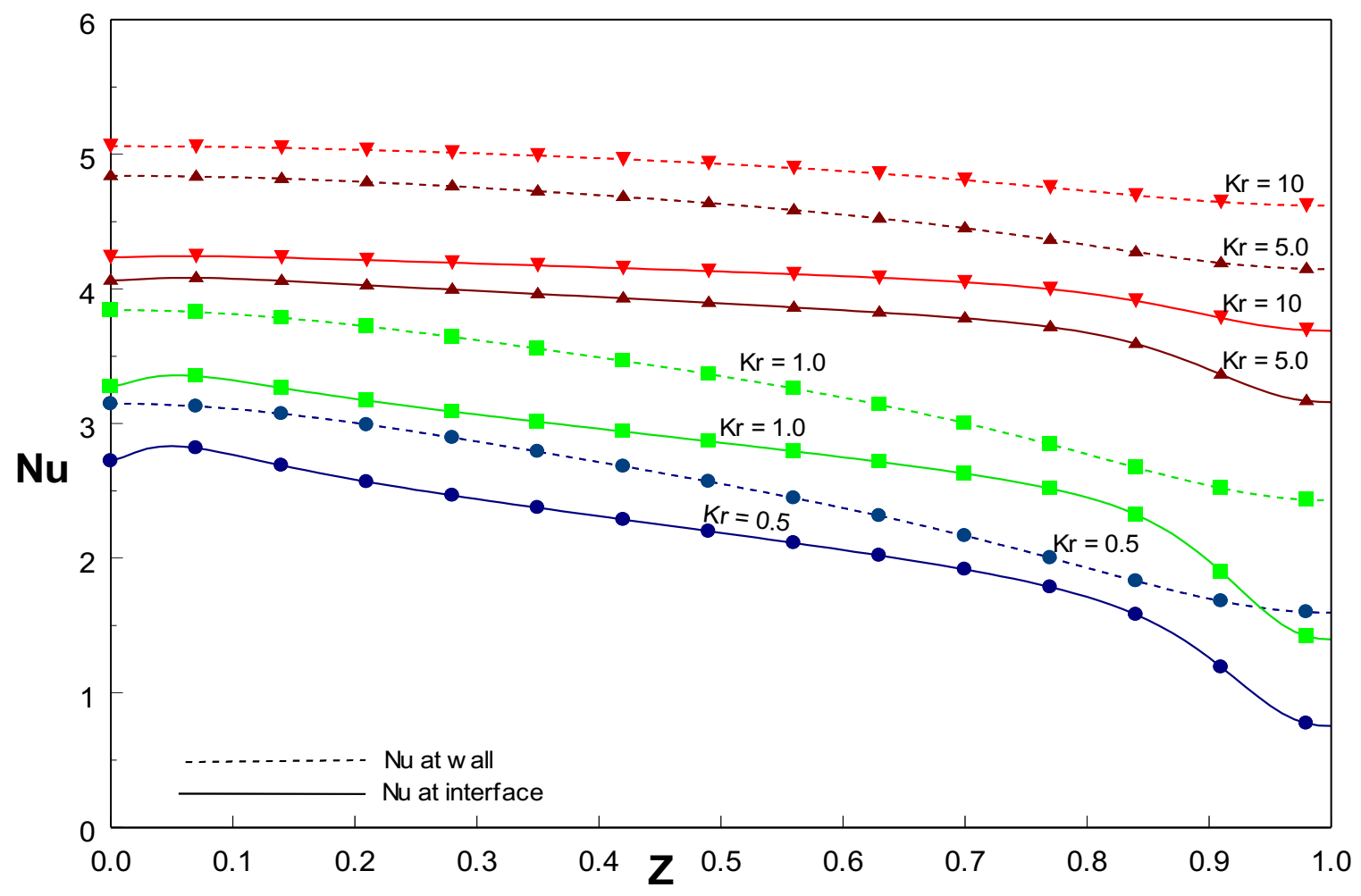

Fig. 10. Effect of $K_{r}$ on the local Nusselt number for $\mathrm{Ra}=10^{6}, \mathrm{Da}=10^{-3}$ and $\boldsymbol{\varepsilon}=0.2$.

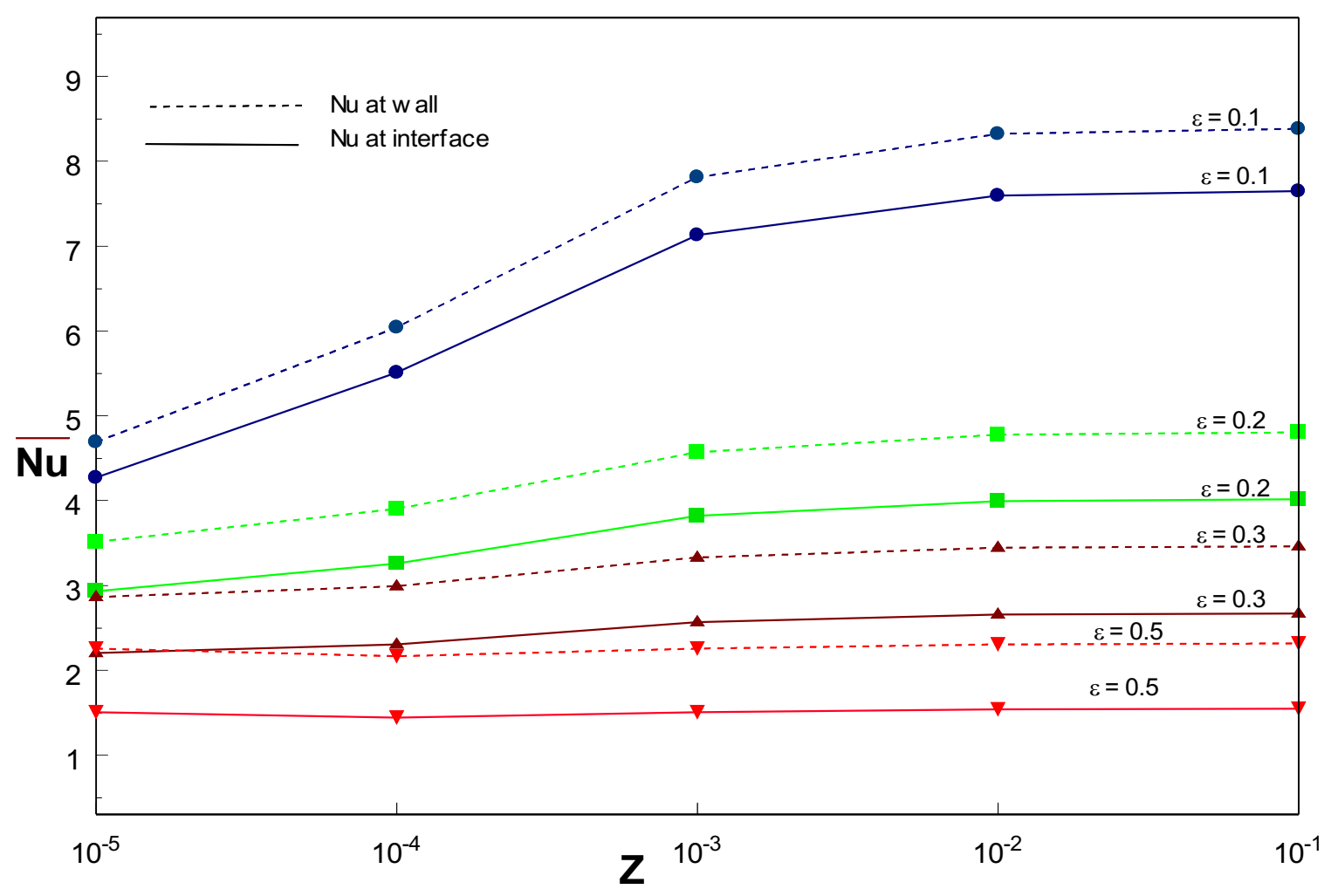

Fig. 11. Effect of Da on average Nusselt number for different $\varepsilon$ at $\mathrm{Ra}=10^{6}$ and $K_{r}=5$. 


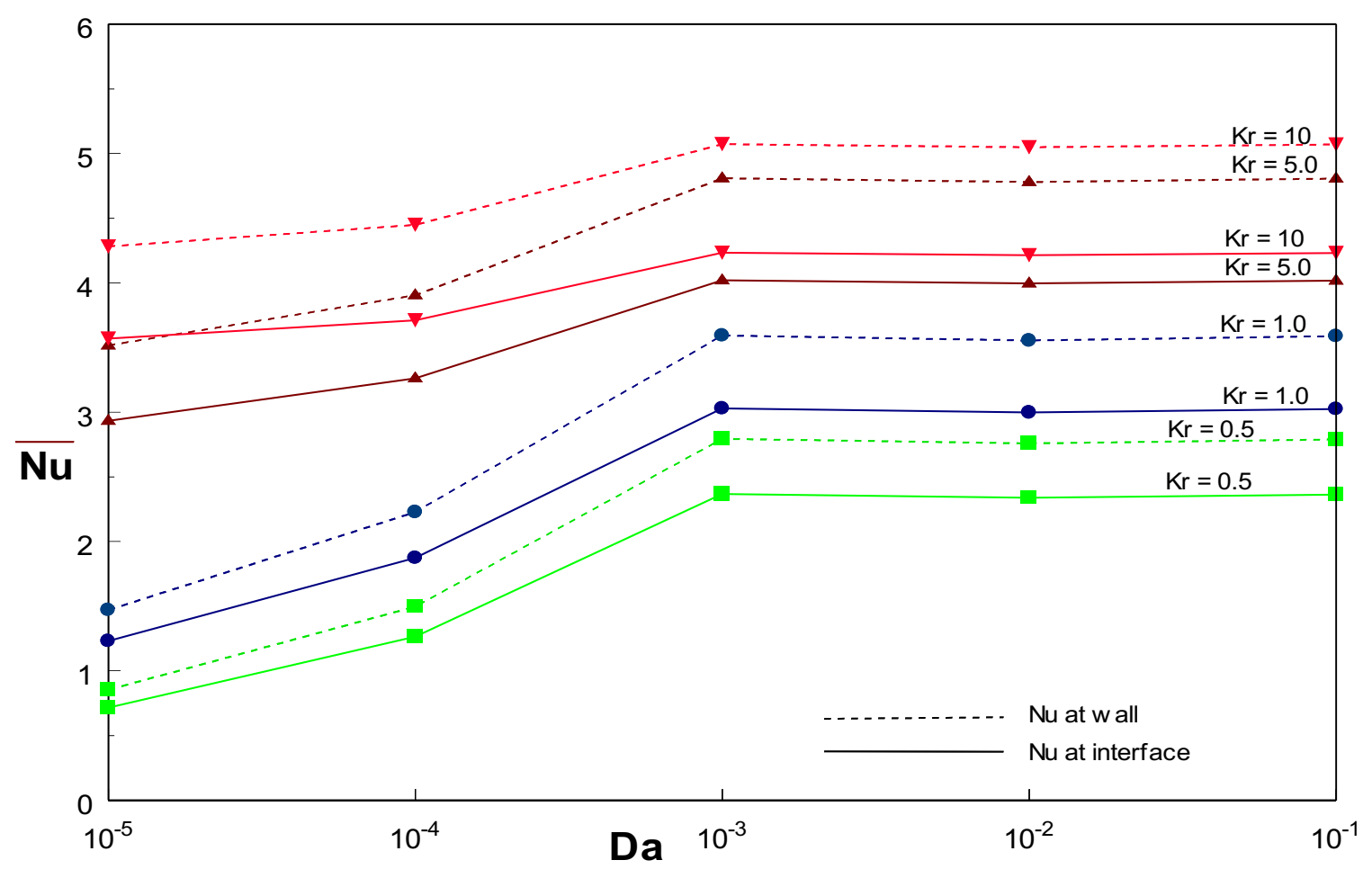

Fig. 12. Effect of $\mathrm{Da}$ on average Nusselt number for different $\mathrm{Kr}$ at $\mathrm{Ra}=10^{6}$ and $\boldsymbol{\varepsilon}=0.2$.

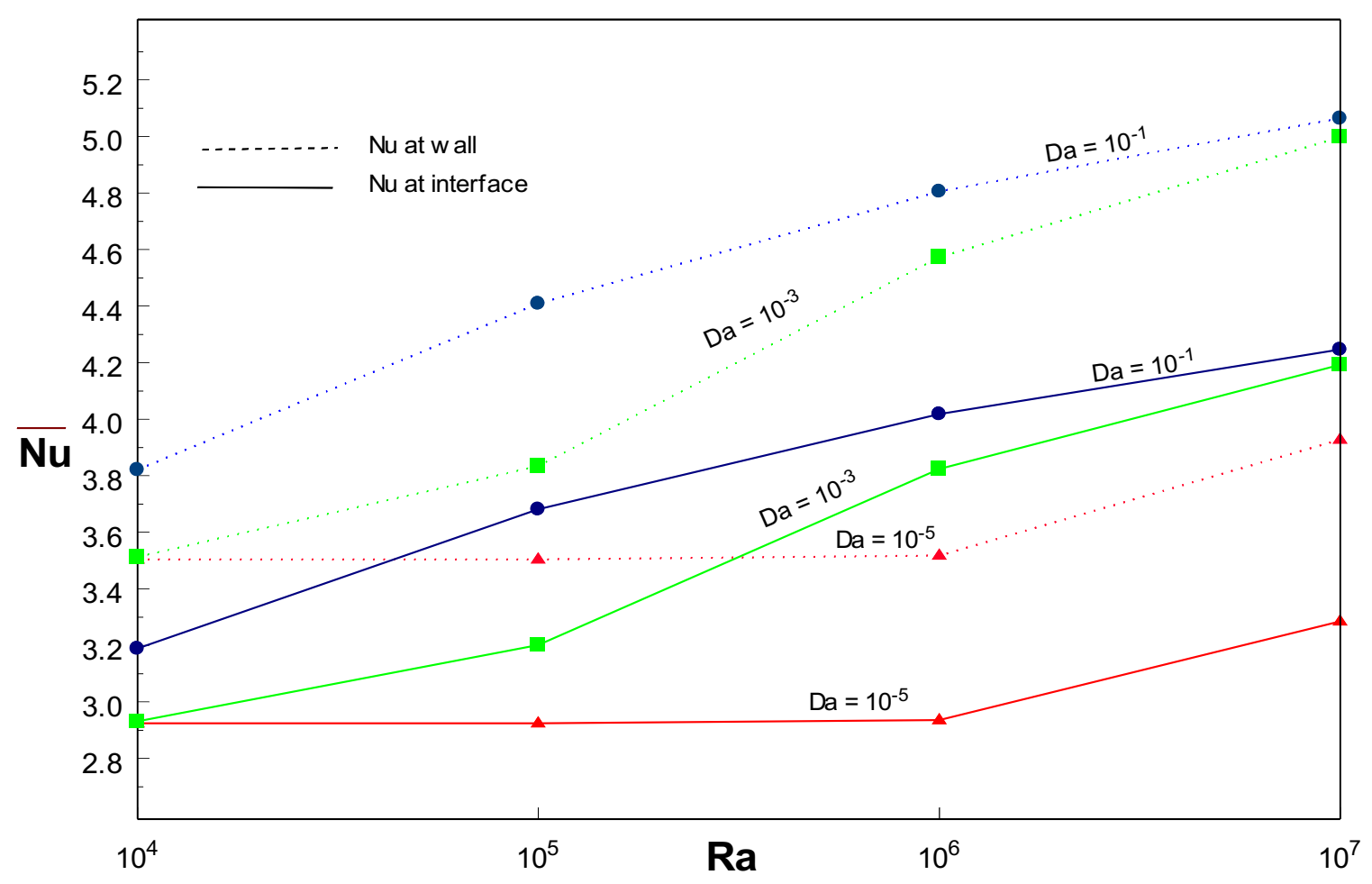

Fig. 13. Effect of Ra and Da on average Nusselt number for $\varepsilon=0.2$ and $K_{r}=5$. 


\section{Conclusions}

In this investigation, conjugate convective heat transport in a porous annular space between vertical, co-axial cylinders. The significant impact of conductivity ratio, inner cylinder-thickness, and Rayleigh number on the convective flow and associated thermal energy transfer has been extensively investigated numerically. It has been established that the thermal conductivity ratio and wall thickness significantly alter the convective stream in the porous annulus. The presence of porous media reduces the convective flow and the resulting thermal transport. Along the wall, the local Nusselt number declines with the axial distance. Moreover, the overall heat energy transport could be improved by raisingraising the conductivity ratio, Rayleigh number, Darcy number or by decreasing the wall thickness. It is worth mentioning that this study is limited to two-dimensional flow with constant fluid properties.

\section{ACKNOWLEDGEMENTS}

"The authors are grateful to the respective Managements and VTU, Belgaum, India. M. Sankar acknowledges the financial support provided by the VGST, GoK, under Grant Number KSTePS/VGST-KFIST (L1)/2017.”

\section{References}

AL-Jawary, M, (2020), Three iterative methods for solving Jeffery-Hamel flow problem, Kuwait Journal of Science, 47 (1): 1-13.

Al-Ashhab, S., (2019), Asymptotic Behaviour and Existence of Similarity Solutions for a Boundary Layer Flow Problem, Kuwait Journal of Science, 46 (2), 13-20.

Ayoubloo, K. A., Ghalambaz, M., Armaghani, T., Noghrehabadi, A. and Chamkha, A.J. (2019), Pseudoplastic natural convection flow and heat transfer in a cylindrical vertical-cavity partially filled with a porous layer, International Journal of Numerical Methods for Heat \& Fluid Flow, 30 (3):1096-1114.

Badruddin, I.A., Al-Rashed, A.A.A.A., Ghazali, N.N., Jameel, M., Kamangar, S., Khaleed, H.M.T., and Khan, T.M.Y. (2015), Conjugate heat transfer in an annulus with porous medium fixed between solids, Transport in Porous Media, 109 (3): 589-608.

Baytas, A.C., Liaqat, A., Grosan, T., and Pop, I. (2001), Conjugate natural convection in a square porous cavity, Heat and Mass Transfer, 37: 467-473. 
Bourouis, A., Omara, A. and Abboudi, S. (2016), Upward and downward conjugate mixed convection heat transfer in a partially porous cavity, International Journal of Numerical Methods for Heat \& Fluid Flow, 26 (1):159-188.

Chamkha, A.J., and Ismael, M.A. (2013), Conjugate heat transfer in a porous cavity heated by a thick triangular wall, Numerical Heat Transfer, Part A: Application, 63 (2): 144-158.

Chung, K.H., Kwak, H.S., and Hyun, J.M. (2001), Finite-wall effect on buoyant convection in an enclosure with pulsating exterior surface temperature, International Journal for Heat and Mass Transfer, 44 (4): 721-732.

De Vahl Davis, G, Thomas, R.W. (1969), Natural convection between concentric vertical cylinders, Physics of Fluids, Supplement II, 12: 198-207.

Ghalambaz, M., Mehryan, S.A.M., Ismael, M.A., Chamkha, A. and Wen, D. (2019), Fluidstructure interaction of free convection in a square cavity divided by a flexible membrane and subjected to sinusoidal temperature heating, International Journal of Numerical Methods for Heat \& Fluid Flow, 30 (6): 2883-2911.

Hasnaoui, M., Vasseur, P., Bilgen, E., and Robillard, L. (1995), Analytical and numerical study of natural convection heat transfer in a vertical porous annulus, Chem. Eng. Commun, 131: 141159.

Havstad, M.A., and Burns, P.J. (1982), Convective heat transfer in vertical cylindrical annuli filled with a porous medium", International Journal for Heat and Mass Transfer, 25 (11): 17551766.

Hdhiri, N. and Ben Beya, B. (2018), Numerical study of laminar mixed convection flow in a liddriven square cavity filled with porous media: Darcy-Brinkman-Forchheimer and DarcyBrinkman models", International Journal of Numerical Methods for Heat \& Fluid Flow, 28 (4): 857-877.

Hickox, C.E., and Gartling, D.K. (1985), A numerical study of natural convection in a vertical, annular, porous layer, International Journal for Heat and Mass Transfer, 28 (3): 720-723.

Kaminski, D.A., and Prakash, C. (1986), Conjugate natural convection in a square enclosure: effect of conduction in one of the vertical walls", International Journal for Heat and Mass Transfer, 29 (12): 1979-1988. 
Kim, D.M., and Viskanta, R. (1984), Study of the effects of wall conductance on natural convection in differently oriented square cavities, Journal of Fluid Mechanics, 144: 153-176.

Kim, D.M., and Viskanta, R. (1985), Effect of wall heat conduction on natural convection heat transfer in a square enclosure, ASME Journal of Heat Transfer, 107 (4): 139-146.

Kiwan, S. and Zeitoun, O. (2008), Natural convection in a horizontal cylindrical annulus using porous fins", International Journal of Numerical Methods for Heat \& Fluid Flow, 18 (5): 618-634.

Kuznetsov, G.V., and Sheremet, M.A. (2011), Conjugate natural convection in an enclosure with a heat source of constant heat transfer rate, International Journal for Heat and Mass Transfer, 54 (1): $260-268$.

Lopez, J.M., Sankar, M., and Do, Y. (2012), Constant-flux discrete heating in a unit aspect-ratio annulus, Fluid Dynamics Research, 44 (6): 18.

Massarotti, N., Ciccolella, M., Cortellessa, G. and Mauro, A. (2016), New benchmark solutions for transient natural convection in partially porous annuli, International Journal of Numerical Methods for Heat \& Fluid Flow, 26 (3/4): 1187-1225.

Mbaye, M., Bilgen, E., and Vasseur, P. (1993), Natural-convection heat transfers in an inclined porous layer boarded by a finite-thickness wall, International Journal for Heat and Fluid Flow, 14 (3): $284-291$.

Mebarek-Oudina, F. (2017), Numerical modelling of the hydrodynamic stability in vertical annulus with a heat source of different lengths. Engineering Science \& Technology an International Journal, 20: 1324-1333

Nazarafkan, H., Mehmandoust, B., Toghraie, D. and Karimipour, A. (2019), Numerical study of natural convection of nanofluid in a semi-circular cavity with lattice Boltzmann method, International Journal of Numerical Methods for Heat \& Fluid Flow, 30 (5): 2625-2637.

Nithiarasu P, Seetharamu K N, Sundararajan T. (1997), Non-Darcy double-diffusive natural convection in axisymmetric fluid-saturated porous cavities, Heat and Mass Transfer, 32: 427433.

Oztop, H.F., Sun, C., and Yu, B. (2008), Conjugate-mixed convection heat transfer in a lid-driven enclosure with thick bottom wall, International Communications in Heat and Mass Transfer, 35 (6): 779-785. 
Prasad, V. (1986), Numerical study of natural convection in a vertical, porous annulus with constant heat flux on the inner wall, International Journal for Heat and Mass Transfer, 29: 841853.

Prasad, V., and Kulacki, F.A. (1984), Natural convection in a vertical porous annulus, International Journal for Heat and Mass Transfer, 27 (2): 207-219.

Saeid, N. H., (2007), Conjugate natural convection in a vertical porous layer sandwiched by finite thickness walls, International Communications in Heat and Mass Transfer, 34 (2): 210-216.

Sankar, M., and Do, Y. (2010), Numerical simulation of free convection heat transfer in a vertical annular cavity with discrete heating, International. Journal for. Heat and Mass Transfer, 37(6): 600-606.

Sankar, M., Bhuvaneswari, M., Sivasankaran, S., and Do, Y. (2011), Buoyancy induced convection in a porous cavity with partially thermally active sidewalls, International Journal for Heat and Mass Transfer, 54 (25-26): 5173-5182.

Sankar, M., Do, Y., Ryu, S., and Jang, B., (2015), Cooling of heat sources by natural convection heat transfer in a vertical annulus, Numerical Heat Transfer, Part A: Application, 68 (8): 847-869.

Sankar, M., Park, J., Kim, D., and Do, Y. (2013), Numerical study of natural convection in a vertical porous annulus with an internal heat source: effect of discrete heating, Numerical Heat Transfer, Part A: Applications, 63 (9): 687-712.

Sankar, M., Park, Y., Lopez, J.M., and Do, Y. (2011), Numerical study of natural convection in a vertical porous annulus with discrete heating, International Journal for Heat and Mass Transfer, 54 (7-8): 1493-1505.

Sheremet, M.A. (2012), Laminar natural convection in an inclined cylindrical enclosure having finite thickness walls, International Journal for Heat and Mass Transfer, 55 (13-14): 3582-3600.

Shivakumara, I.S., Prasanna, B.M.R., Rudraiah, N., and Venkatachalappa, M. (2002), Numerical study of natural convection in a vertical cylindrical annulus using a non-Darcy equation, Journal of Porous Media, 5: 87-102.

Sivasankaran, S, and Bhuvaneswari, M. (2013), Natural convection in a porous cavity with sinusoidal heating on both sidewalls. Numerical Heat Transfer, Part A: Applications, 63: 14-30. 
Tahmasebi, A., Mahdavi, M., and Ghalambaz, M. (2018), Local thermal nonequilibrium conjugate natural convection heat transfer of nanofluids in a cavity partially filled with porous media using Buongiorno's model, Numerical Heat Transfer, Part A: Applications, 73 (4): 254276.

Venkatachalappa, M., Sankar, M., and Natarajan, A.A. (2001), Natural convection in an annulus between two rotating vertical cylinders, Acta Mechanica, 147 (1-4): 173-196.

Wu, F., Zhou, W., and Ma, X. (2015), Natural convection in a porous rectangular enclosure with sinusoidal temperature distributions on both side walls using a thermal non-equilibrium model, International Journal for Heat and Mass Transfer, 85: 756-771.

Zadeh, S.M.H., Sabour, M., Sazgara, S., and Galambaz, M., (2019), Free convection flow and heat transfer of nanofluids in a cavity with conjugate solid triangular blocks: Employing Buongiorno's mathematical model, Physica A: Statistical Mechanics and Its Applications, 538: 122826.

Submitted: $\quad 22 / 10 / 2020$

Revised: $\quad 06 / 02 / 2021$

Accepted: 28/03/2021

DOI: $\quad 10.48129 /$ kjs.10789 